\title{
A MODIFIED STATE VARIABLE DIAGRAM METHOD FOR DETERMINATION OF POSITIVE REALIZATIONS OF LINEAR CONTINUOUS-TIME SYSTEMS WITH DELAYS
}

\author{
TADEUSZ KACZOREK \\ Faculty of Electrical Engineering \\ Białystok Technical University, ul. Wiejska 45D, 15-351 Białystok, Poland \\ e-mail:kaczorek@isep.pw.edu.pl
}

\begin{abstract}
A new modified state variable diagram method is proposed for determination of positive realizations of linear continuoustime systems with delays in state and input vectors. Using the method, it is possible to find a positive realization with reduced numbers of delays for a given transfer matrix. Sufficient conditions for the existence of positive realizations of given proper transfer matrices are established. The proposed method is demonstrated on numerical examples.
\end{abstract}

Keywords: state diagram method, determination, linear, continuous-time, delay, realization.

\section{Introduction}

Determination of the state space equations for a given transfer matrix is a classical problem, called the realization problem, which has been addressed in many papers and books (Farina and Rinaldi, 2000; Benvenuti and Farina, 2004; Kaczorek, 1992; 2008; 2011d; 2012a; 2012b; Shaker and Dixon, 1977). It is well known (Farina and Rinaldi, 2000; Kaczorek, 2002; 1992) that to find a realization for a given transfer function first we have to find a state matrix for given denominator of the transfer function.

Some overviews on the positive realization problem are given by Farina and Rinaldi (2000), Kaczorek (2002), as well as Benvenuti and Farina (2004). The realization problem for positive continuous-time and discretetime linear systems was considered by Kaczorek (2006a; 2006b; 2011a; 2011b; 2006c; 2004; 2011c) along with the positive realization problem for discrete-time systems with delays (Kaczorek, 2006; 2004; 2005). Fractional positive linear systems were addressed by Kaczorek (2008c; 2009a; 2011d), together with the realization problem for fractional linear systems (Kaczorek, 2008a) and for positive 2D hybrid systems (Kaczorek, 2008b). A method based on a similarity transformation of the standard realization to the discrete positive one was proposed by Kaczorek (2011c). Conditions for the existence of a positive stable realization with a system Metzler matrix for transfer function were established by Kaczorek (2011a), who also formulated and solved the problem of determination of the set of Metzler matrices for given stable polynomials (Kaczorek, 2012a).

In this paper a new modified state variable diagram method for determination of positive realizations with a reduced number of delays for given proper transfer matrices will be proposed and sufficient conditions for the existence of positive realizations will be established. The proposed method allows us to find a positive realization with the number of delays less than the one that follows from the degree of the denominator of a given transfer function.

The paper is organized as follows. In Section 2 some preliminaries concerning positive continuous-time linear systems with delays are recalled and the problem formulation is given. Basic lemmas of the proposed method are given in Section 3. The new modified state variable diagram method is proposed in Section 4. Concluding remarks are given in Section 5

The following notation will be used: $\mathbb{R}$ is the set of real numbers, $\mathbb{R}^{n \times m}$ is the set of $n \times m$ real matrices, $\mathbb{R}_{+}^{n \times m}$ is the set of $n \times m$ matrices with nonnegative entries and $\mathbb{R}_{+}^{n}=\mathbb{R}_{+}^{n \times 1}, M_{n}$ is the set of $n \times n$ Metzler matrices (real matrices with nonnegative off-diagonal entries), $I_{n}$ is the $n \times n$ identity matrix, $A^{T}$ is the transpose of a matrix $A, \mathbb{R}^{n \times m}(s, w)$ is the set of $n \times m$ rational matrices in $s$ and $w, \mathbb{R}^{n \times m}[s, w]$ is the set of $n \times m$ polynomial matrices in $s$ and $w$. 


\section{Preliminaries and problem formulation}

Consider the continuous-time linear system with $h$ delays in state and $q$ delays in inputs,

$$
\begin{aligned}
& \dot{x}(t)=\sum_{i=0}^{h} A_{i} x(t-i d)+\sum_{j=0}^{q} B_{j} u(t-j d), \\
& y(t)=C x(t)+D u(t),
\end{aligned}
$$

where $x(t) \in \mathbb{R}^{n}, u(t) \in \mathbb{R}^{m}, y(t) \in \mathbb{R}^{p}$ are the state, input and output vectors, respectively, and $A_{i} \in \mathbb{R}^{n \times n}$, $i=0,1, \ldots, h, B_{j} \in \mathbb{R}^{n \times m}, j=0,1, \ldots, q, C \in \mathbb{R}^{p \times n}$, $D \in \mathbb{R}^{p \times m}, d>0$ being a delay.

Initial conditions for (1) are given by

$$
\begin{aligned}
& x_{0}(t) \text { for } t \in[-h d, 0], \\
& u_{0}(t) \text { for } t \in[-q d, 0] .
\end{aligned}
$$

Definition 1. The system (1) is called (internally) positive for every $x_{0}(t) \in \mathbb{R}_{+}^{n}, t \in[-h d, 0]$ if $u_{0}(t) \in \mathbb{R}_{+}^{m}, t \in$ $[-q d, 0]$ and all inputs $u(t) \in \mathbb{R}_{+}^{m}, t \geq 0$ we have $x(t) \in$ $\mathbb{R}_{+}^{n}$ and $y(t) \in \mathbb{R}_{+}^{p}$ for $t \geq 0$.

Theorem 1. (Kaczorek, 2005) The system (1) is positive if and only if

$$
\begin{aligned}
& A_{0} \in M_{n}, A_{i} \in \mathbb{R}_{+}^{n \times n}, \quad i=1,2, \ldots, h, \\
& B_{j} \in \mathbb{R}_{+}^{n \times m}, \quad j=0,1, \ldots, q, \\
& C \in \mathbb{R}_{+}^{p \times n}, \quad D \in \mathbb{R}_{+}^{p \times m} .
\end{aligned}
$$

The transfer matrix of the system (1) is given by

$$
\begin{aligned}
T(s, w)= & C\left[I_{n} s-A_{0}-A_{1} w-\cdots-A_{h} w^{h}\right]^{-1} \\
& {\left[B_{0}+B_{1} w+\cdots+B_{q} w^{q}\right]+D, } \\
w= & e^{-d s} .
\end{aligned}
$$

Definition 2. The matrices (3) are called a positive realization of a given transfer matrix $T(s, w) \in \mathbb{R}^{p \times m}(s, w)$ if they satisfy (4).

The positive realization problem under consideration can be stated as follows: Given a proper transfer matrix $T(s, w) \in \mathbb{R}^{p \times m}(s, w)$, find a positive realization with reduced numbers of delays (3) of $T(s, w)$.

In this paper sufficient conditions for the problem solvability will be established and a new method for determination of a positive realization with a reduced number of delays will be proposed.

\section{Problem solution}

The transfer matrix (4) can be written in the following form:

$$
\begin{aligned}
& T(s, w) \\
& =\frac{C\left(H_{a d}(s, w)\right)\left[B_{0}+B_{1} w+\cdots+B_{q} w^{q}\right]}{\operatorname{det} H(s, w)}+D \\
& =\frac{N(s, w)}{d(s, w)}+D
\end{aligned}
$$

where

$$
\begin{aligned}
& H(s, w) \\
& =\left[I_{n} s-A_{0}-A_{1} w-\cdots-A_{h} w^{h}\right] \in \mathbb{R}^{p \times m}[s, w],
\end{aligned}
$$

$$
\begin{aligned}
& N(s, w)=C\left(H_{a d}(s, w)\right)\left[B_{0}+B_{1} w+\cdots+B_{q} w^{q}\right], \\
& d(s, w)=\operatorname{det} H(s, w) .
\end{aligned}
$$

From (5) we have

$$
D=\lim _{s \rightarrow \infty} T(s, w)
$$

since $\lim _{s \rightarrow \infty} H^{-1}(s, w)=0$. The strictly proper transfer matrix is given by

$$
T_{s p}(s, w)=T(s, w)-D=\frac{N(s, w)}{d(s, w)} .
$$

Therefore, the positive realization problem is reduced to finding the matrices

$$
\begin{aligned}
& A_{0} \in M_{n}, \quad A_{i} \in \mathbb{R}_{+}^{n \times n}, \quad i=1,2, \ldots, h, \\
& B_{j} \in \mathbb{R}_{+}^{n \times m}, \quad j=0,1, \ldots, q, \quad C \in \mathbb{R}_{+}^{p \times n},
\end{aligned}
$$

for a given strictly proper transfer matrix (9).

To simplify the notation, we shall consider a SingleInput Single-Output (SISO) system described by (1) for $m=p=1$.

Let a given, strictly proper, irreducible transfer function have the form

$$
\begin{aligned}
T_{s p}(s, w)= & \frac{n(s, w)}{d(s, w)} \\
n(s, w)= & b_{n-1}(w) s^{n-1}+\cdots+b_{1}(w) s+b_{0}(w) \\
b_{k}(w)= & b_{k, m} w^{m}+\cdots+b_{k, 1} w+b_{k, 0} \\
& k=0,1, \ldots, n-1 \\
d(s, w)= & s^{n}-a_{n-1}(w) s^{n-1}-\ldots \\
& -a_{1}(w) s-a_{0}(w) \\
a_{k}(w)= & a_{k, m} w^{m}+\cdots+a_{k, 1} w+a_{k, 0} \\
& \quad k=0,1, \ldots, n-1
\end{aligned}
$$

The solution of the positive realization problem for (11) is based on the following two lemmas (Kaczorek, 2006a).

Lemma 1. Let $p_{k}=p_{k}(w)$ for $k=1,2, \ldots, 2 n-1$ be some polynomials in $w$ with nonnegative coefficients and

$$
P(w)=\left[\begin{array}{ccccc}
0 & 0 & \cdots & 0 & p_{n} \\
p_{1} & 0 & \cdots & 0 & p_{n+1} \\
0 & p_{2} & \cdots & 0 & p_{n+2} \\
\vdots & \vdots & \cdots & \vdots & \vdots \\
0 & 0 & \cdots & p_{n-1} & p_{2 n-1}
\end{array}\right] .
$$


Then

$$
\begin{aligned}
\operatorname{det} & {\left[I_{n} s-P(w)\right] } \\
= & s^{n}-p_{2 n-1} s^{n-1}-p_{n-1} p_{2 n-2} s^{n-2}-\ldots \\
& \quad-p_{2} p_{3} \ldots p_{n-1} p_{n+1} s-p_{1} p_{2} \ldots p_{n} .
\end{aligned}
$$

The proof is given by Kaczorek (2006a).

Lemma 2. Let $R_{n}(w)$ be the $n$-th row of the adjoint matrix $\left[I_{n} s-P(w)\right]_{a d}$. Then

$$
\begin{aligned}
& R_{n}(w) \\
& =\left[\begin{array}{lllllll}
p_{1} p_{2} \ldots p_{n-1} & p_{2} p_{3} & \ldots & p_{n-1} s & p_{3} p_{4} & \ldots & p_{n-1} s^{2}
\end{array}\right. \\
& \left.\ldots p_{n-1} s^{n-2} s^{n-1}\right] \text {. }
\end{aligned}
$$

The proof is given by Kaczorek (2006a).

From Lemmas 1 and 2 we obtain that, if

$$
P(w)=\left[\begin{array}{ccccc}
0 & 0 & \ldots & 0 & p_{2} \\
p_{1} & 0 & \ldots & 0 & p_{3} \\
0 & p_{1} & \ldots & 0 & p_{4} \\
\vdots & \vdots & \ldots & \vdots & \vdots \\
0 & 0 & \ldots & p_{1} & p_{n+1}
\end{array}\right]
$$

then

$$
\begin{aligned}
& \operatorname{det}\left[I_{n} s-P(w)\right] \\
& =s^{n}-p_{n+1} s^{n-1}-\cdots-p_{3} p_{1}^{n-2} s-p_{2} p_{1}^{n-1},
\end{aligned}
$$

and

$$
R_{n}(s)=\left[\begin{array}{lllll}
p_{1}^{n-1} & p_{1}^{n-2} s & \ldots & p_{1} s^{n-2} & s^{n-1}
\end{array}\right] .
$$

It is assumed that for a given denominator (11c) there exist polynomials

$$
\begin{aligned}
p_{k}=p_{k}(w)=p_{k, h} w^{h}+\cdots & +p_{k, 1} w+p_{k, 0}, \\
k & =0,1, \ldots, 2 n-1,
\end{aligned}
$$

with nonnegative coefficients $p_{k, j}, j=0,1, \ldots, h$ such that

$$
\begin{aligned}
a_{n-1}(w) & =p_{2 n-1}, \\
a_{n-2}(w) & =p_{n-1} p_{2 n-2}, \ldots, \\
a_{1}(w) & =p_{2} p_{3} \ldots p_{n-1} p_{n+1}, \\
a_{0}(w) & =p_{1} p_{2} \ldots p_{n} .
\end{aligned}
$$

In a particular case, if the matrix $P(w)$ has the form (15), then (19) takes the form

$$
a_{k}(w)=p_{1}^{n-k-1} p_{k+2}, \quad k=0,1, \ldots, n-1 .
$$

Note that if the assumption (19) is satisfied, then for a given denominator $d, s, w)$ of (11a) we may find the matrix (12) and next the corresponding matrices $A_{i} \in \mathbb{R}_{+}^{n \times n}$, $i=1,2, \ldots, h$, since

$$
I_{n} s-P(w)=I_{n} s-\sum_{i=0}^{h} A_{i} w^{i} .
$$

The matrix $C$ is chosen in the form

$$
C=\left[\begin{array}{llll}
0 & \ldots & 0 & 1
\end{array}\right] \in \mathbb{R}^{1 \times n} .
$$

Taking into account (14), (21) and (7), we obtain

$$
\begin{aligned}
& C\left[I_{n} s-P(w)\right]_{a d}\left[B_{0}+B_{1} w+\cdots+B_{q} w^{q}\right] \\
& =R_{n}(w)\left[B_{0}+B_{1} w+\cdots+B_{q} w^{q}\right] \\
& =\left[\begin{array}{lll}
p_{1} p_{2} \ldots p_{n-1} & p_{2} p_{3} \ldots p_{n-1} s & p_{3} p_{4} \ldots p_{n-1} s^{2}
\end{array}\right. \\
& \left.\ldots p_{n-1} s^{n-2} s^{n-1}\right] \\
& {\left[B_{0}+B_{1} w+\cdots+B_{q} w^{q}\right]} \\
& =n(s, w) \text {. }
\end{aligned}
$$

\section{Modified state variables diagram method}

First the modified state variables diagram method of determination of positive realizations is presented on the strictly proper transfer function (24).

The proposed method is based on Lemmas 1 and 2 . It is assumed that there exist polynomials (18) with nonnegative coefficients $p_{k, j}, j=0,1, \ldots, h$, (except the last coefficient $p_{k, 0}$ ) satisfying (19) and

$$
\begin{aligned}
b_{n-1}(w) & =\bar{b}_{n-1}(w), \\
b_{n-2}(w) & =p_{n-1} \bar{b}_{n-2}(w), \\
& \vdots \\
b_{1}(w) & =p_{2} p_{3} \ldots p_{n-1} \bar{b}_{1}(w), \\
b_{0}(w) & =p_{1} p_{2} \ldots p_{n-1} \bar{b}_{0}(w),
\end{aligned}
$$

for some polynomials with nonnegative coefficients $\bar{b}_{n-2}(w), \ldots, \bar{b}_{1}(w), \bar{b}_{0}(w)$.

For (24) we have

$p_{1}(w)=w^{2}, \quad p_{2}(w)=w+1, \quad p_{3}(w)=w^{2}+w+2$, $p_{4}(w)=w^{2}+2 w, \quad p_{5}(w)=2 w^{2}+3 w-1$,

since

$$
\begin{aligned}
& \operatorname{det}\left[I_{3} s-P(w)\right] \\
&=\left|\begin{array}{ccc}
s & 0 & -w^{2}-w-2 \\
-w^{2} & s & -w^{2}-2 w \\
0 & -w-1 & s-2 w^{2}-3 w+1
\end{array}\right| \\
&= s^{3}-\left(2 w^{2}+3 w-1\right) s^{2} \\
&-\left(w^{3}+3 w^{2}+2 w\right) s \\
&-\left(w^{5}+2 w^{4}+3 w^{3}+2 w^{2}\right) \\
&= s^{3}-a_{2}(w) s^{2}-a_{1}(w) s-a_{0}(w), \\
& a_{2}(w)= p_{5}(w), \quad a_{1}(w)=p_{2}(w) p_{4}(w), \\
& a_{0}(w)=p_{1}(w) p_{2}(w) p_{3}(w),
\end{aligned}
$$




$$
\begin{aligned}
T_{s p}(s, w) & =\frac{b_{2}(w) s^{2}+b_{1}(w) s+b_{0}(w)}{s^{3}-a_{2}(w) s^{2}-a_{1}(w) s-a_{0}(w)} \\
& =\frac{\left(3 w^{2}+w+2\right) s^{2}+\left(w^{2}+3 w+2\right) s+w^{4}+2 w^{3}+w^{2}}{s^{3}-\left(2 w^{2}+3 w-1\right) s^{2}-\left(w^{3}+3 w^{2}+2 w\right) s-\left(w^{5}+2 w^{4}+3 w^{3}+2 w^{2}\right)}
\end{aligned}
$$

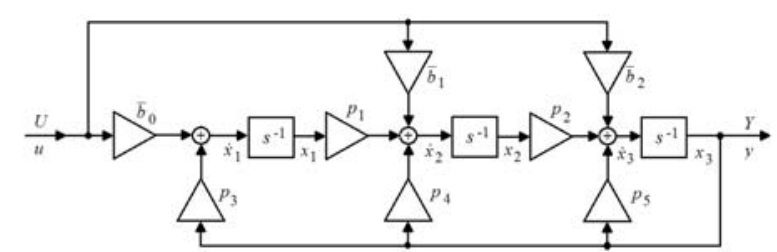

Fig. 1. State variable diagram for (28).

and

$$
\begin{aligned}
b_{2}(w) & =3 w^{2}+w+2, \quad b_{1}(w)=p_{2}(w) \\
\bar{b}_{1}(w) & =(w+1)(w+2)=w^{2}+3 w+2 \\
b_{0}(w) & =p_{1}(w) p_{2}(w) \bar{b}_{0}(w) \\
& =\left(w^{3}+w^{2}\right)(w+1)=w^{4}+2 w^{3}+w^{2}
\end{aligned}
$$

where

$$
\bar{b}_{1}(w)=w+2, \quad \bar{b}_{0}(w)=w+1 .
$$

From (24) written in the form

$$
\frac{b_{2}(w) s^{-1}+b_{1}(w) s^{-2}+b_{0}(w) s^{-3}}{1-a_{2}(w) s^{-1}-a_{1}(w) s^{-2}-a_{0}(w) s^{-3}}=\frac{Y}{U},
$$

we have

$$
\begin{aligned}
Y= & s^{-1}\left\{b_{2}(w) U+a_{2}(w) Y+s^{-1}\left[b_{1}(w) U\right.\right. \\
& \left.\left.+a_{1}(w) Y+s^{-1}\left(b_{0}(w) U+a_{0}(w) Y\right)\right]\right\} \\
= & s^{-1}\left\{\bar{b}_{2}(w) U+p_{5}(w) Y+s^{-1}\left[p_{2}(w) \bar{b}_{1} U\right.\right. \\
& +p_{2}(w) p_{4}(w) Y+s^{-1}\left(p_{1}(w) p_{2}(w) \bar{b}_{0}(w) U\right. \\
& \left.\left.\left.+p_{1}(w) p_{2}(w) p_{3}(w) Y\right)\right]\right\} .
\end{aligned}
$$

Using (28) we may draw the modified state variable diagram shown in Fig. 1. The variables $x_{1}, x_{2}, x_{3}$ are chosen as the outputs of the integral elements. Using the modified state variables diagram, we can write the following equations:

$$
\begin{aligned}
& \dot{x}_{1}=p_{3} x_{3}+\bar{b}_{0} u, \\
& \dot{x}_{2}=p_{1} x_{1}+p_{4} x_{3}+\bar{b}_{1} u, \\
& \dot{x}_{3}=p_{2} x_{2}+p_{5} x_{3}+\bar{b}_{2} u,
\end{aligned}
$$

which can be also written in the form

$$
\dot{x}=A x+B u,
$$

where

$$
\begin{aligned}
A & =\left[\begin{array}{ccc}
0 & 0 & p_{3} \\
p_{1} & 0 & p_{4} \\
0 & p_{2} & p_{5}
\end{array}\right]=P(w)=A_{0}+A_{1} w+A_{2} w^{2}, \\
A_{0} & =\left[\begin{array}{ccc}
0 & 0 & 2 \\
0 & 0 & 0 \\
0 & 1 & -1
\end{array}\right], \quad A_{1}=\left[\begin{array}{lll}
0 & 0 & 1 \\
0 & 0 & 2 \\
0 & 1 & 3
\end{array}\right], \\
A_{2} & =\left[\begin{array}{lll}
0 & 0 & 1 \\
1 & 0 & 1 \\
0 & 0 & 2
\end{array}\right], \quad B=\left[\begin{array}{l}
\bar{b}_{0} \\
\bar{b}_{1} \\
\bar{b}_{2}
\end{array}\right] .
\end{aligned}
$$

Assuming $C=\left[\begin{array}{lll}0 & 0 & 1\end{array}\right]$ and taking into account (29b), we have

$$
\begin{aligned}
& T_{s p}(s, w)=C\left[I_{3} s-A\right]^{-1} B \\
& =\left[\begin{array}{lll}
0 & 0 & 1
\end{array}\right]\left[\begin{array}{ccc}
s & 0 & -p_{3} \\
-p_{1} & s & -p_{4} \\
0 & -p_{2} & s-p_{5}
\end{array}\right]^{-1}\left[\begin{array}{l}
\bar{b}_{0} \\
\bar{b}_{1} \\
\bar{b}_{2}
\end{array}\right]
\end{aligned}
$$

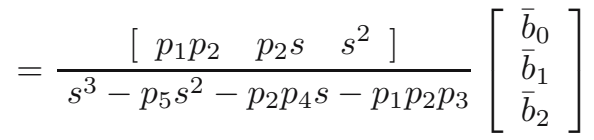

$$
\begin{aligned}
& =\frac{p_{1} p_{2} \bar{b}_{0}+p_{2} \bar{b}_{1} s+\bar{b}_{2} s^{2}}{s^{3}-a_{2}(w) s^{2}-a_{1}(w) s-a_{0}(w)} .
\end{aligned}
$$

Comparison of (30) and (24) yields

$$
\begin{aligned}
& \bar{b}_{2}(w)=b_{2}(w)=3 w^{2}+w+2, \\
& \bar{b}_{1}(w)=w+2, \quad \bar{b}_{0}(w)=w+1
\end{aligned}
$$

and

$$
\bar{B}=\left[\begin{array}{l}
\bar{b}_{0}(w) \\
\bar{b}_{1}(w) \\
\bar{b}_{2}(w)
\end{array}\right]=B_{0}+B_{1} w+B_{2} w^{2},
$$

where

$$
B_{0}=\left[\begin{array}{l}
1 \\
2 \\
2
\end{array}\right], \quad B_{1}=\left[\begin{array}{l}
1 \\
1 \\
1
\end{array}\right], \quad B_{2}=\left[\begin{array}{l}
0 \\
0 \\
3
\end{array}\right] .
$$

The desired positive realization of (24) is given by (29c), (31c) and $C=\left[\begin{array}{lll}0 & 0 & 1\end{array}\right], D=[0]$.

Note that the sufficient condition for the existence of a positive realization of (24) is the existence of the polynomials (26) and $\bar{b}_{1}(w), \bar{b}_{0}(w)$ with nonnegative coefficients satisfying (27a) and (27b). 
In a general case, consider the strictly proper irreducible transfer function

$$
T_{s p}(s, w)=\frac{n(s, w)}{d(s, w)}
$$

where

$$
\begin{aligned}
n(s, w) & =b_{n-1}(w) s^{n-1}+\cdots+b_{1}(w) s+b_{0}(w), \\
b_{k}(w) & =b_{k, m} w^{m}+\cdots+b_{k, 1} w+b_{k, 0}, \\
& k=0,1, \ldots, n-1, \\
d(s, w) & =s^{n}-a_{n-1}(w) s^{n-1}-\cdots-a_{1}(w) s-a_{0}(w), \\
a_{k}(w) & =a_{k, m} w^{m}+\cdots+a_{k, 1} w+a_{k, 0}, \\
& k=0,1, \ldots, n-1 .
\end{aligned}
$$

It is assumed that there exist the polynomials (32) and $\bar{b}_{0}(w), \bar{b}_{1}(w), \ldots, \bar{b}_{n-2}(w)$ with nonnegative coefficients (except the last coefficient of $p_{2 n-1}(w)$ satisfying (25).

By Lemma 1 we have

$$
\begin{aligned}
& \operatorname{det}\left[I_{n} s-P(w)\right] \\
& =\left|\begin{array}{ccccc}
s & 0 & \ldots & 0 & -p_{n} \\
-p_{1} & s & \ldots & 0 & -p_{n+1} \\
0 & -p_{2} & \ldots & 0 & -p_{n+2} \\
\vdots & \vdots & \ldots & \vdots & \vdots \\
0 & 0 & \ldots & -p_{n-1} & s-p_{2 n-1}
\end{array}\right| \\
& =s^{n}-p_{2 n-1} s^{n-1}-p_{n-1} p_{2 n-2} s^{n-2}-\ldots \\
& \\
& -p_{2} p_{3} \ldots p_{n-1} p_{n+1} s-p_{1} p_{2} \ldots p_{n},
\end{aligned}
$$

and, by Lemma 2, the $n$-th row of $R_{n}(w)$ of the adjoint matrix $\left[I_{n} s-P(w)\right]_{a d}$ is

$$
\begin{aligned}
& R_{n}(w) \\
& =\left[\begin{array}{llll}
p_{1} p_{2} \ldots p_{n-1} & p_{2} p_{3} \ldots p_{n-1} s & p_{3} p_{4} \ldots p_{n-1} s^{2} \\
& \ldots & p_{n-1} s^{n-2} & s^{n-1}
\end{array}\right] .
\end{aligned}
$$

Let

$$
\bar{B}(w)=\left[\begin{array}{c}
\bar{b}_{0}(w) \\
\vdots \\
\bar{b}_{n-1}(w)
\end{array}\right]=\left[\begin{array}{c}
\bar{b}_{0} \\
\vdots \\
\bar{b}_{n-1}
\end{array}\right]
$$

and

$$
\begin{aligned}
R_{n}(w) & \bar{B}(w) \\
= & p_{1} p_{2} \ldots p_{n-1} \bar{b}_{0}+p_{2} p_{3} \ldots p_{n-1} \bar{b}_{1} s+\ldots \\
& +p_{n-1} \bar{b}_{n-2} s^{n-2}+\bar{b}_{n-1} s^{n-1} \\
= & b_{0}+b_{1} s+\ldots+b_{n-2} s^{n-2}+b_{n-1} s^{n-1} \\
= & n(s, w) .
\end{aligned}
$$

Assuming $C=\left[\begin{array}{llll}0 & \ldots & 0 & 1\end{array}\right] \in \mathbb{R}_{+}^{1 \times n}$ and using (33), (36), we obtain

$$
\begin{aligned}
& T_{s p}(s, w)=C\left[I_{n} s-A(w)\right]^{-1} \bar{B}(w) \\
& =\left[\begin{array}{lllll}
0 & \ldots & 0 & 1
\end{array}\right] \\
& {\left[\begin{array}{ccccc}
s & 0 & \ldots & 0 & -p_{n} \\
-p_{1} & s & \ldots & 0 & -p_{n+1} \\
0 & -p_{2} & \ldots & 0 & -p_{n+2} \\
\vdots & \vdots & \ldots & \vdots & \vdots \\
0 & 0 & \ldots & -p_{n-1} & s-p_{2 n-1}
\end{array}\right]\left[\begin{array}{c}
\bar{b}_{0} \\
\vdots \\
\bar{b}_{n-1}
\end{array}\right]} \\
& =\frac{R_{n}(w) \bar{B}(w)}{s^{n}-a_{n-1}(w) s^{n-1}-\ldots-a_{1}(w) s-a_{0}(w)} \\
& =\frac{n(s, w)}{d(s, w)} .
\end{aligned}
$$

Theorem 2. A positive realization

$$
\begin{aligned}
A_{0} \in M_{n}, \quad A_{i} \in \mathbb{R}_{+}^{n \times n}, \quad i=1,2, \ldots, h \\
B_{j} \in \mathbb{R}_{+}^{n \times m}, \quad j=0,1, \ldots, q, \\
C=\left[\begin{array}{llll}
0 & \ldots & 0 & 1
\end{array}\right] \in \mathbb{R}_{+}^{1 \times n}
\end{aligned}
$$

of the transfer function (32) exists if it is possible to find polynomials

$$
p_{1}(w), \quad p_{2}(w), \ldots, \quad p_{2 n-1}(w)
$$

and

$$
\bar{b}_{0}(w), \quad \bar{b}_{1}(w), \ldots, \quad \bar{b}_{n-1}(w)
$$

with nonnegative coefficients (except the last coefficient of $p_{2 n-1}(w)$ such that (33) and (36) are satisfied.

Proof. If the polynomials (39) have nonnegative coefficients (except the last coefficient of $p_{2 n-1}(w)$ ), then

$$
A(w)=P(w)=A_{h} w^{h}+\cdots+A_{1} w+A_{0}
$$

and $A_{0} \in M_{n}, A_{i} \in \mathbb{R}_{+}^{n \times n}, i=1,2, \ldots, h$. If the coefficients of the polynomials (40) are nonnegative, then

$$
\bar{B}(w)=\left[\begin{array}{c}
\bar{b}_{0}(w) \\
\vdots \\
\bar{b}_{n-1}(w)
\end{array}\right]=B_{q} w^{q}+\cdots+B_{1} w+B_{0}
$$

and $B_{j} \in \mathbb{R}_{+}^{n \times m}, j=0,1, \ldots, q$. The matrices (38) are a realization of (32) since they satisfy (37).

If the assumption of Theorem 2 is met, then the positive realization (38) of (32) can be found by using the following procedure.

\section{Procedure 1.}

Step 1. Knowing the coefficients $a_{k}(w), b_{k}(w), k=$ $0,1, \ldots, n-1$ of (32), find the polynomials (39) and 
(40) with nonnegative coefficients satisfying the conditions (33) and (36).

Step 2. Knowing (39) and using (41), find the matrices $A_{k}$ for $k=0,1, \ldots, h$.

Step 3. Using the equalities 36 and (42), find the polynomials $\bar{b}_{0}(w), \bar{b}_{1}(w), \ldots, \bar{b}_{n-1}(w)$ and the matrices $B_{j}$, $j=0,1, \ldots, q$.

Example 1. Using Procedure 1, find a positive realization of the transfer function

$$
T_{s p}(s, w)=\frac{\left(w^{2}+2 w\right) s+\left(w^{3}+w^{2}\right)}{s^{2}-(2 w-3) s-\left(w^{3}+w\right)} .
$$

Step 1 . In this case we choose the polynomials

$$
p_{1}(w)=w, \quad p_{2}(w)=w^{2}+1, \quad p_{3}(w)=2 w-3
$$

and

$$
\bar{b}_{0}(w)=w^{2}+w, \quad \bar{b}_{1}(w)=w^{2}+2 w,
$$

which satisfy the conditions (33) and (36) since

$$
\begin{aligned}
& \operatorname{det}\left[I_{2} s-P(w)\right]=\left|\begin{array}{cc}
s & -p_{2} \\
-p_{1} & s-p_{3}
\end{array}\right| \\
& =s^{2}-p_{3} s-p_{1} p_{2}=s^{2}-(2 w-3) s-\left(w^{3}+w\right)
\end{aligned}
$$

and

$$
\begin{aligned}
{\left[\begin{array}{ll}
p_{1} & s
\end{array}\right]\left[\begin{array}{l}
\bar{b}_{0}(w) \\
\bar{b}_{1}(w)
\end{array}\right] } & =\left[\begin{array}{ll}
w & s
\end{array}\right]\left[\begin{array}{c}
w^{2}+w \\
w^{2}+2 w
\end{array}\right] \\
& =\left(w^{2}+2 w\right) s+\left(w^{3}+w^{2}\right)
\end{aligned}
$$

Step 2. Using (41) and (44), we obtain

$A(w)=P(w)=\left[\begin{array}{cc}0 & w^{2}+1 \\ w & 2 w-3\end{array}\right]=A_{0}+A_{1} w+A_{2} w^{2}$

where

$$
\begin{aligned}
& A_{0}=\left[\begin{array}{cc}
0 & 1 \\
0 & -3
\end{array}\right], \quad A_{1}=\left[\begin{array}{ll}
0 & 0 \\
1 & 2
\end{array}\right], \\
& A_{2}=\left[\begin{array}{ll}
0 & 1 \\
0 & 0
\end{array}\right] .
\end{aligned}
$$

Step 3. Taking into account that

$$
\begin{aligned}
\bar{B}(w) & =\left[\begin{array}{l}
\bar{b}_{0}(w) \\
\bar{b}_{1}(w)
\end{array}\right]=\left[\begin{array}{c}
w^{2}+w \\
w^{2}+2 w
\end{array}\right] \\
& =B_{0}+B_{1} w+B_{2} w^{2}
\end{aligned}
$$

where

$$
B_{0}=\left[\begin{array}{l}
0 \\
0
\end{array}\right], \quad B_{1}=\left[\begin{array}{l}
1 \\
2
\end{array}\right], \quad B_{2}=\left[\begin{array}{l}
1 \\
1
\end{array}\right],
$$

the desired positive realization of (43) is given by (48), (4.26b) and

$$
C=\left[\begin{array}{ll}
0 & 1
\end{array}\right], \quad D=[0]
$$

The proposed method can be extended to a MultiInput Multi-Output (MIMO) linear system with delays.

It is well known that the proper transfer matrix of MIMO linear systems with delays can be written in the form

$$
\begin{aligned}
T(s, w)= & {\left[\begin{array}{ccc}
\frac{n_{11}(s, w)}{d_{1}(s, w)} & \ldots & \frac{n_{1, m}(s, w)}{d_{1}(s, w)} \\
\vdots & \ldots & \vdots \\
\frac{n_{p, 1}(s, w)}{d_{p}(s, w)} & \cdots & \frac{n_{p, m}(s, w)}{d_{p}(s, w)}
\end{array}\right] } \\
& +D \in \mathbb{R}^{p \times m}(s, z),
\end{aligned}
$$

where

$$
\begin{gathered}
n_{i, j}(s, w)=b_{n_{i}-1}^{i, j}(w) s^{n_{i}-1}+\cdots+b_{1}^{i, j}(w) s+b_{0}^{i, j}(w), \\
\quad i=1,2, \ldots, \quad p, j=1,2, \ldots, m, \\
b_{k}^{i, j}(w)=b_{k, q_{i}}^{i, j} w^{q_{i}}+\cdots+b_{k, 1}^{i, j} w+b_{k, 0}^{i, j}, \\
k=0,1, \ldots, n_{i}-1 .
\end{gathered}
$$

Theorem 3. A positive realization

$$
\begin{gathered}
A_{0}=\operatorname{blockdiag}\left[A_{01}, \ldots, A_{0, p}\right] \in M_{n}, \\
n=n_{1}+\cdots+n_{p}, \\
A_{k}=\operatorname{blockdiag}\left[A_{k, 1}, \ldots, A_{k, p}\right] \in \mathbb{R}_{+}^{n \times n}, \\
k=1,2, \ldots, h, \\
B_{k}=\left[\begin{array}{ccc}
B_{11}^{k} & \ldots & B_{1, m}^{k} \\
\vdots & \ldots & \vdots \\
B_{p, 1}^{k} & \cdots & B_{p, m}^{k}
\end{array}\right] \in \mathbb{R}_{+}^{n \times m}, \\
k=0,1, \ldots, q,
\end{gathered}
$$

$$
\begin{aligned}
C & =\text { blockdiag }\left[C_{1}, \ldots, C_{p}\right] \in \mathbb{R}_{+}^{p \times n}, \\
C_{i} & =\left[\begin{array}{llll}
0 & \ldots & 0 & 1
\end{array}\right] \in \mathbb{R}_{+}^{1 \times n_{i}}, \quad i=1,2, \ldots, p,
\end{aligned}
$$

of the strictly proper transfer matrix (50) if it is possible to find the polynomials

$$
p_{1}^{i}(w), p_{2}^{i}(w), \ldots, p_{2 n_{i}-1}^{i}(w), \quad i=1,2, \ldots, p
$$

and

$$
\begin{aligned}
& \bar{b}_{0}^{i, j}(w), \bar{b}_{1}^{i, j}(w), \ldots, \bar{b}_{n_{i}-1}^{i, j}(w), \\
& \quad i=1,2, \ldots, p, \quad j=1,2, \ldots, m
\end{aligned}
$$


with nonnegative coefficients (except the last coefficient of $\left.p_{2 n_{i}-1}^{i}(w)\right)$ such that the conditions

$$
\begin{aligned}
& \operatorname{det}\left[I_{n_{i}} s-P_{i}(w)\right] \\
& =\left|\begin{array}{ccccc}
s & 0 & \ldots & 0 & -p_{n_{i}}^{i} \\
-p_{1}^{i} & s & \ldots & 0 & -p_{n_{i}+1} \\
0 & -p_{2}^{i} & \ldots & 0 & -p_{n_{i}+2}^{i} \\
\vdots & \vdots & \ldots & \vdots & \vdots \\
0 & 0 & \ldots & -p_{n_{i}-1}^{i} & s-p_{2 n_{i}-1}^{i}
\end{array}\right| \\
& =d_{i}(s, w), \quad i=1,2, \ldots, p, \\
& p_{1}^{i} p_{2}^{i} \ldots p_{n_{i}-1}^{i} \bar{b}_{0}^{i, j}+p_{2}^{i} p_{3}^{i} \ldots p_{n_{i}-1}^{i} \bar{b}_{1}^{i, j} s \\
& +\cdots+p_{n_{i}-1}^{i} \bar{b}_{n_{i}-2}^{i, j} s^{n_{i}-2}+\bar{b}_{n_{i}-1}^{i, j} s^{n_{i}-1} \\
& =b_{0}^{i, j}+b_{1}^{i, j} s+\cdots+b_{n_{i}-2}^{i, j} s^{n_{i}-2}+b_{n_{i}-1}^{i, j} s^{n_{i}-1} \\
& =n_{i, j}(s, w), \\
& \\
& i=1,2, \ldots, p, \quad j=1,2, \ldots, m,
\end{aligned}
$$

are satisfied.

Proof. If the polynomials (52a) have nonnegative coefficients (except the last coefficient of $p_{2 n_{i}-1}^{i}$ ), then

$$
\begin{array}{r}
A_{k}(w)=P_{k}(w)=\operatorname{blockdiag}\left[A_{k, 1}, \ldots, A_{k, p}\right], \\
k=0,1, \ldots, h,
\end{array}
$$

and (51a) and (51b) hold. If the coefficients of the polynomials (52b) are nonnegative, then

$$
\begin{aligned}
B_{i, j}(w)=B_{i, j}^{0}+B_{i, j}^{1} w+\cdots+B_{i, j}^{q} w^{q}, \\
\quad i=1,2, \ldots, p, \quad j=1,2, \ldots, m,
\end{aligned}
$$

and $(51 \mathrm{c})$ is satisfied. It is easy to check that the matrices (51) and $D \in \mathbb{R}_{+}^{p \times m}$ satisfy the equality

$$
C\left[I_{n} s-A\right]^{-1} B+D=T(s, w),
$$

where $T(s, w)$ is given by (50a).

If the conditions of Theorem 3 are satisfied, then the positive realization of (50a) can be found by using the following procedure.

\section{Procedure 2.}

Step 1. Using the formula

$$
D=\lim _{s \rightarrow \infty} T(s, w),
$$

find the matrix $D$ and the strictly proper part $T_{s p}(s, w)$ of (50a).

Step 2. Choose the polynomials (52) with nonnegative coefficients (except the last coefficient of $p_{2 n_{i}-1}^{i}$ ) satisfying (53) and (54).

Step 3. Using (55), find the matrices (51a) and (51b).

Step 4. Using (56), find the matrices (51c) and (51d).
Example 2. Find a positive realization of the strictly proper transfer matrix

$$
T_{s p}(s, w)=\left[\begin{array}{ll}
T_{11} & T_{12} \\
T_{21} & T_{22}
\end{array}\right],
$$

where $T_{11}, T_{12}, T_{21}$ and $T_{22}$ are given by (58b).

Using Procedure 2, we obtain the following:

Step 1. The transfer matrix (58) already has the desired form (50) with $D=0$.

Step 2. For the first row of (58), we choose

$$
\begin{aligned}
& p_{1}^{1}=w^{2}, \quad p_{2}^{1}=w+1, \quad p_{3}^{1}=w+2, \\
& p_{4}^{1}=w, \quad p_{5}^{1}=w^{2}-3,
\end{aligned}
$$

and for the second row,

$$
p_{1}^{2}=w^{2}+1, \quad p_{2}^{2}=w+1, \quad p_{3}^{2}=w^{2}-2,
$$

since

$$
\operatorname{det}\left[I_{3} s-P_{1}(w)\right]=\left|\begin{array}{ccc}
s & 0 & -(w+2) \\
-w^{2} & s & -w \\
0 & -(w+1) & s-w^{2}+3
\end{array}\right|
$$$$
=s^{3}-\left(w^{2}-3\right) s^{2}-\left(w^{2}+w\right) s-\left(w^{4}+3 w^{3}+2 w^{2}\right) \text {, }
$$

$\operatorname{det}\left[I_{2} s-P_{2}(w)\right]=\left|\begin{array}{cc}s & -(w+1) \\ -\left(w^{2}+1\right) & s-w^{2}+2\end{array}\right|$

$=s^{2}-\left(w^{2}-2\right) s-\left(w^{3}+w^{2}+w+1\right)$

and

$$
\begin{aligned}
& {\left[\begin{array}{lll}
p_{1}^{1} p_{2}^{1} & p_{2}^{1} s & s^{2}
\end{array}\right]\left[\begin{array}{c}
\bar{b}_{0}^{1,1}(w) \\
\bar{b}_{1}^{1,1}(w) \\
\bar{b}_{2}^{1,1}(w)
\end{array}\right]} \\
& \quad=\left[\begin{array}{lll}
w^{3}+w^{2} & (w+1) s & s^{2}
\end{array}\right]\left[\begin{array}{c}
1 \\
w \\
w^{2}+2
\end{array}\right] \\
& \quad=\left(w^{2}+2\right) s^{2}+\left(w^{2}+w\right) s+w^{3}+w^{2},
\end{aligned}
$$

$\left[\begin{array}{lll}p_{1}^{1} p_{2}^{1} & p_{2}^{1} s & s^{2}\end{array}\right]\left[\begin{array}{l}\bar{b}_{0}^{1,2}(w) \\ \bar{b}_{1}^{1,2}(w) \\ \bar{b}_{2}^{1,2}(w)\end{array}\right]$

$$
\begin{aligned}
& =\left[\begin{array}{lll}
w^{3}+w^{2} & (w+1) s & s^{2}
\end{array}\right]\left[\begin{array}{c}
2 w \\
w^{2} \\
w^{3}+w
\end{array}\right] \\
& =\left(w^{3}+w\right) s^{2}+\left(w^{3}+w^{2}\right) s+2 w^{4}+2 w^{3},
\end{aligned}
$$

$$
\begin{aligned}
& {\left[\begin{array}{ll}
p_{1}^{2} & s
\end{array}\right]\left[\begin{array}{l}
\bar{b}_{0}^{2,1}(w) \\
\bar{b}_{1}^{2,1}(w)
\end{array}\right]=\left[\begin{array}{ll}
w^{2}+1 & s
\end{array}\right]\left[\begin{array}{c}
w \\
w^{2}+2
\end{array}\right]} \\
& =\left(w^{2}+2\right) s+w^{3}+w \\
& {\left[\begin{array}{ll}
p_{1}^{2} & s
\end{array}\right]\left[\begin{array}{c}
\bar{b}_{0}^{2,2}(w) \\
\bar{b}_{1}^{2,2}(w)
\end{array}\right]=\left[\begin{array}{ll}
w^{2}+1 & s
\end{array}\right]\left[\begin{array}{c}
w+1 \\
w^{3}
\end{array}\right]} \\
& =w^{3} s+w^{3}+w^{2}+w+1 .
\end{aligned}
$$




$$
\begin{aligned}
& T_{11}=\frac{\left(w^{2}+2\right) s^{2}+\left(w^{2}+w\right) s+w^{3}+w^{2}}{s^{3}-\left(w^{2}-3\right) s^{2}-\left(w^{2}+w\right) s-\left(w^{4}+3 w^{3}+2 w^{2}\right)}, \\
& T_{12}=\frac{\left(w^{3}+w\right) s^{2}+\left(w^{3}+w^{2}\right) s+2 w^{4}+2 w^{3}}{s^{3}-\left(w^{2}-3\right) s^{2}-\left(w^{2}+w\right) s-\left(w^{4}+3 w^{3}+2 w^{2}\right)}, \\
& T_{21}=\frac{\left(w^{2}+2\right) s+w^{3}+w}{s^{2}-\left(w^{2}-2\right) s-\left(w^{3}+w^{2}+w+1\right)}, \\
& T_{22}=\frac{w^{3} s+w^{3}+w^{2}+w+1}{s^{2}-\left(w^{2}-2\right) s-\left(w^{3}+w^{2}+w+1\right)} .
\end{aligned}
$$

Step 3. Using (55) and (59), we obtain

$$
\begin{gathered}
A_{1}(w)=P_{1}(w)=A_{10}+A_{11} w+A_{12} w^{2} \\
A_{10}=\left[\begin{array}{ccc}
0 & 0 & 2 \\
0 & 0 & 0 \\
0 & 1 & -3
\end{array}\right], \quad A_{11}=\left[\begin{array}{lll}
0 & 0 & 1 \\
0 & 0 & 1 \\
0 & 1 & 0
\end{array}\right] \\
A_{12}=\left[\begin{array}{lll}
0 & 0 & 0 \\
1 & 0 & 0 \\
0 & 0 & 1
\end{array}\right] \\
A_{2}(w)=P_{2}(w)=A_{20}+A_{21} w+A_{22} w^{2} \\
A_{20}=\left[\begin{array}{cc}
0 & 1 \\
1 & -2
\end{array}\right], A_{21}=\left[\begin{array}{cc}
0 & 1 \\
0 & 0
\end{array}\right] \\
A_{22}=\left[\begin{array}{cc}
0 & 0 \\
1 & 1
\end{array}\right]
\end{gathered}
$$

and

$$
A_{k}=\operatorname{blockdiag}\left[A_{k, 1}, A_{k, 2}\right], \quad k=0,1,2 .
$$

Step 4. Using (56), (61) and (62), we obtain

$$
\begin{aligned}
& \bar{B}_{11}(w)= {\left[\begin{array}{c}
1 \\
w \\
w^{2}+2
\end{array}\right]=B_{11}^{0}+B_{11}^{1} w+B_{11}^{2} w^{2} } \\
& B_{11}^{0}= {\left[\begin{array}{l}
1 \\
0 \\
2
\end{array}\right], \quad B_{11}^{1}=\left[\begin{array}{l}
0 \\
1 \\
0
\end{array}\right] } \\
& B_{11}^{2}=\left[\begin{array}{l}
0 \\
0 \\
1
\end{array}\right], \quad B_{11}^{3}=\left[\begin{array}{l}
0 \\
0 \\
0
\end{array}\right]
\end{aligned}
$$

$$
\begin{aligned}
& \bar{B}_{12}(w)= {\left[\begin{array}{c}
2 w \\
w^{2} \\
w^{3}+w
\end{array}\right] } \\
&= B_{12}^{0}+B_{12}^{1} w+B_{12}^{2} w^{2}+B_{12}^{3} w^{3}, \\
& B_{12}^{0}= {\left[\begin{array}{l}
0 \\
0 \\
0
\end{array}\right], \quad B_{12}^{1}=\left[\begin{array}{l}
2 \\
0 \\
1
\end{array}\right], } \\
& B_{12}^{2}=\left[\begin{array}{l}
0 \\
1 \\
0
\end{array}\right], \quad B_{12}^{3}=\left[\begin{array}{l}
0 \\
0 \\
1
\end{array}\right],
\end{aligned}
$$$$
\bar{B}_{21}(w)=\left[\begin{array}{c}
w \\
w^{2}+2
\end{array}\right]=B_{21}^{0}+B_{21}^{1} w+B_{21}^{2} w^{2},
$$$$
B_{21}^{0}=\left[\begin{array}{l}
0 \\
2
\end{array}\right], \quad B_{21}^{1}=\left[\begin{array}{l}
1 \\
0
\end{array}\right],
$$$$
B_{21}^{2}=\left[\begin{array}{l}
0 \\
1
\end{array}\right], \quad B_{21}^{3}=\left[\begin{array}{l}
0 \\
0
\end{array}\right] \text {, }
$$$$
\bar{B}_{22}(w)=\left[\begin{array}{c}
w+1 \\
w^{3}
\end{array}\right]
$$$$
=B_{22}^{0}+B_{22}^{1} w+B_{22}^{2} w^{2}+B_{22}^{3} w^{3},
$$$$
B_{22}^{0}=\left[\begin{array}{l}
1 \\
0
\end{array}\right], \quad B_{22}^{1}=\left[\begin{array}{l}
1 \\
0
\end{array}\right] \text {, }
$$$$
B_{22}^{2}=\left[\begin{array}{l}
0 \\
0
\end{array}\right], \quad B_{22}^{3}=\left[\begin{array}{l}
0 \\
1
\end{array}\right] \text {, }
$$$$
B_{k}=\left[\begin{array}{ll}
B_{11}^{k} & B_{12}^{k} \\
B_{21}^{k} & B_{22}^{k}
\end{array}\right], \quad k=0,1,2,3 .
$$

The desired positive realization of (58) is given by 64), 66) and

$$
C=\left[\begin{array}{lllll}
0 & 0 & 1 & 0 & 0 \\
0 & 0 & 0 & 0 & 1
\end{array}\right], \quad D=\left[\begin{array}{ll}
0 & 0 \\
0 & 0
\end{array}\right] .
$$

\section{Concluding remarks}

(65a) 
systems with delays in state and input vectors has been proposed. Using the method it is possible to find a positive realization with reduced numbers of delays for given proper transfer matrices. Sufficient conditions for the existence of positive realizations have been established and procedures for finding the positive realizations have been proposed. The procedures have been illustrated by numerical examples. The proposed method can be extended to continuous-discrete linear systems and to fractional continuous-time linear ones.

\section{Acknowledgment}

This work was supported by the National Science Centre in Poland under the grant no. S/WE/1/11.

\section{References}

Benvenuti L. and Farina L. (2004). A tutorial on the positive realization problem, IEEE Transactions on Automatic Control 49(5): 651-664.

Farina L. and Rinaldi S. (2000). Positive Linear Systems, Theory and Applications, J. Wiley, New York, NY.

Kaczorek T. (1992). Linear Control Systems, Vol.1, Research Studies Press, J. Wiley, New York, NY.

Kaczorek T. (2002). Positive $1 D$ and 2D Systems, SpringerVerlag, London.

Kaczorek T. (2004). Realization problem for positive discretetime systems with delay, System Science 30(4): 117-130.

Kaczorek T. (2005). Positive minimal realizations for singular discrete-time systems with delays in state and delays in control, Bulletin of the Polish Academy of Sciences: Technical Sciences 53(3): 293-298.

Kaczorek T. (2006a). A realization problem for positive continuous-time linear systems with reduced numbers of delays, International Journal of Applied Mathematics and Computer Science 16(3): 325-331.

Kaczorek T. (2006b). Computation of realizations of discretetime cone systems, Bulletin of the Polish Academy of Sciences: Technical Sciences 54(3): 347-350.

Kaczorek T. (2006c). Realization problem for positive multivariable discrete-time linear systems with delays in the state vector and inputs, International Journal of Applied Mathematics and Computer Science 16(2): 169-174.

Kaczorek T. (2008a). Realization problem for fractional continuous-time systems, Archives of Control Sciences 18(1): 43-58.

Kaczorek T. (2008b). Realization problem for positive 2D hybrid systems, COMPEL 27(3): 613-623.

Kaczorek T. (2008c). Fractional positive continuous-time linear systems and their reachability, International Journal of Applied Mathematics and Computer Science 18(2): 223-228, DOI: 10.2478/v10006-008-0020-0.

Kaczorek T. (2009a). Fractional positive linear systems, Kybernetes: The International Journal of Systems \& Cybernetics 38(7/8): 1059-1078.
Kaczorek T. (2009b). Polynomial and Rational Matrices, Springer-Verlag, London.

Kaczorek T. (2011a). Computation of positive stable realizations for linear continuous-time systems, Bulletin of the Polish Academy of Sciences: Technical Sciences 59(3): 273-281 and Proceedings of the 20th European Conference on Circuit Theory and Design, Linköping, Sweden.

Kaczorek T. (2011b). Positive stable realizations of fractional continuous-time linear systems, International Journal of Applied Mathematics and Computer Science 21(4): 697702, DOI: 10.2478/v10006-011-0055-5.

Kaczorek T. (2011c). Positive stable realizations with system Metzler matrices, Archives of Control Sciences 21(2): 167188 and Proceedings of the MMAR'2011 Conference, Miedzyzdroje, Poland, (on CD-ROM).

Kaczorek T. (2011d). Selected Problems in Fractional Systems Theory, Springer-Verlag, London.

Kaczorek T. (2012a). Existence and determination of the set of Metzler matrices for given stable polynomials, International Journal of Applied Mathematics and Computer Science 22(2): 389-399, DOI: 10.2478/v10006-012-0029-2.

Kaczorek T. (2012b). Positive stable realizations of discrete-time linear systems, Bulletin of the Polish Academy of Sciences: Technical Sciences 60(3): 605-616.

Shaker U. and Dixon M. (1977). Generalized minimal realization of transfer-function matrices, International Journal of Control 25(5): 785-803.

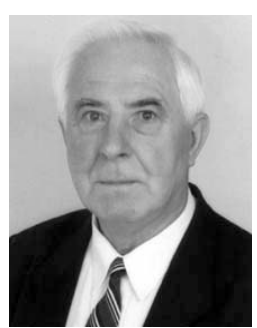

Tadeusz Kaczorek received the M.Sc., Ph.D. and D.Sc. degrees in electrical engineering from the Warsaw University of Technology in 1956, 1962 and 1964, respectively. In the years 1968-69 he was the dean of the Electrical Engineering Faculty, and in the period of 1970-73 he was a deputy rector of the Warsaw University of Technology. In 1971 he became a professor and in 1974 a full professor at the same university. Since 2003 he has been a professor at Białystok Technical University. In 1986 he was elected a corresponding member and in 1996 a full member of the Polish Academy of Sciences. In the years 1988-1991 he was the director of the Research Centre of the Polish Academy of Sciences in Rome. In 2004 he was elected an honorary member of the Hungarian Academy of Sciences. He has been granted honorary doctorates by nine universities. His research interests cover systems theory, especially singular multidimensional systems, positive multidimensional systems, singular positive 1D and 2D systems, as well as positive fractional $1 \mathrm{D}$ and $2 \mathrm{D}$ systems. He initiated research in the field of singular 2D and positive 2D systems. He has published 24 books (six in English) and over 1000 scientific papers. He has also supervised $69 \mathrm{Ph} . \mathrm{D}$. theses. He is the editor-in-chief of the Bulletin of the Polish Academy of Sciences: Technical Sciences and a member of editorial boards of ten international journals.

Received: 1 March 2012 Revised: 24 April 2012 